\title{
Electromagnetic Pair Production in Relativistic Heavy-Ion Collisions
}

CONF-881151--25

DE89 002224

\author{
C. Bottcher and M.R. Strayer \\ Physics Division, Oak Ridge National Laboratory, \\ Oak Ridge, TN 37831, USA
}

October 4, 1988

\section{DISCLAIMER}

\begin{abstract}
This report was prepared as an account of work sponsored by an agency of the United States Government. Neither the United States Government nor any agency thereof, nor any of their employees, makes any warranty, express or implied, or assumes any legal liability or responsibility for the accuracy, completeness, or usefulness of any information, apparatus, product, or process disclosed, or represents that its use would not infringe privatel: owned rights. Reference herein to any specific commercial product, process. or service by trade name, trademark, manufacturer, or otherwise does not necessarily constitute or imply its endorsement, recommendation, or favoring by the United States Government or any agency thereof. The views and opinions of authors expressed herein do not necessarily state or reflect those of the United States Government or any agency thereof.
\end{abstract}

PACS numbers: $11.15 . \mathrm{Kc}, 13.10 .+\mathrm{q}, 13.60 .-\mathrm{r}, 13.86 . \mathrm{Qk}, 14.60 . \mathrm{Cd}, 25.70 . \mathrm{Np}, 34.90 .+\mathrm{q}$

Key Words: Lepton Pairs, Positrons, Muons, Tauons, Relativistic Heavy-Ion Collisions, Two Photon Diagrams, Nuclear Form Factors.

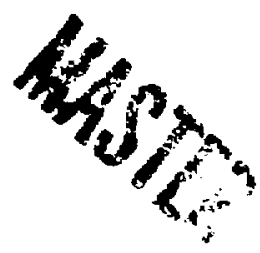




\section{Abstract}

We survey the production of electron, muon and tauon pairs in collisions between $\mathbf{n}$ at ultra-relativistic energies. Such studies enhance our understanding of the role of vacuum in field theory, and provide essential input for several experimentai programs variety of models for the nuclear and nucleon form factors have been considered, revea some degree of sensitivity to assumptions about sub-nuclear structure. We predict that cross sections, even at high invariant masses and transverse momenta, are large on hadr scales, and should act as useful probes of nuclear and nucleon form factors. 


\section{Introduction}

The production of lepton pairs by purely electromagnetic means, in the intense shortlived fields produced by the collisions of highly stripped heavy ions, has been a subject of intense interest for almost two decades. Remarkably, interest is still growing, particularly in ultra-high collision energies, well above one Gev per nucleon $[1,2,3,4]$. Our earlier work, mostly at lower energies, was surveyed in a recent article [5].

These processes are of fundamental interest as an uncluttered test bench for the so called gauge theories of particle interactions. While we believe that such theories describe most of the physical world, we cannot calculate them except in perturbative limits, or using the immensely complicated lattice gauge techniques, which are still in their infancy. However, the strong fields in heavy ion collisions can be treated classically, offering the possibility of a breakthrough into non-perturbative regimes with less effort.

Leptonic pair production also impinges on current and proposed experimental programs. In the Relativistic Heavy Ion Collider (RHIC) facility to be constructed at Brookhaven, the lifetime of the merged beams is limited by capture following pair production. It is therefore desirable to have a theory, calibrated by at least one experiment, in advance of completing RHIC. Ambitious efforts are in train to identify the postulated quark-gluon plasma by detecting di-lepton pairs $[6,7,8,9]$. Theory is needed to distinguish plasma pairs from electromagnetic pairs, which will be copiously produced $[10,11,12]$.

In the present paper, we draw back from the program of non-perturbative formulations, to survey the information which can be gleaned from perturbation theory. While serious objections have been raised to the use of perturbation theory, we adopt the view in this paper as in Refs. $[13,14]$, that exact second-order Feynmann perturbation theory can be used as a heuristic tool. The emphasis on exact theory, in contrast to such formulations as that of Weizsäcker and Williams, is vital. Our calculations in this framework have shown that electron- positron pairs are produced in significant numbers at large pair masses and transverse momenta, where virtual photon approximations are invalid.

The remainder of this paper is divided as follows. Section 2 summarizes the exact formu- 
lation of perturbation theory. Section 3 deals with some useful analytic simplifications. In Section 4 we apply our methods to electron, muon and tauon pair production, and to capture of electrons. Section 5 summarizes some conclusions and points out future directions.

\section{Exact Formulations of Inclusive Electromagnetic Pair Production}

Consider a collision between two bare ions $a$ and $b$, viewed in a frame such that $a$ moves from left to right at a velocity $+\beta$ parallel to the $z$-axis, while $b$ moves from right to left at a velocity $-\beta$ parallel to the $z$-axis. The nuclei have charges $Z_{a}$ and $Z_{b}$, and masses $m_{a}$ and $m_{b}$. Their trajectories are separated by an impact parameter, $\vec{\rho}$. We employ natural units defined by $\hbar=c=m=e=1$. Each nucleus has a kinetic energy per nucleon, $E$, related in these units to the Lorentz factor, $\gamma=\left(1-\beta^{2}\right)^{\frac{1}{2}}$, by $\gamma=E+1$.

Suppose the collision produces a lepton pair,

$$
a+b \rightarrow a^{\prime}+b^{\prime}+\ell^{-}(k)+\ell^{+}(q)
$$

We shall mostly consider $a$ and $b$ as rigid charge distributions; simultaneous nuclear excitation can be incorporated in our formulation. In lowest order, (1) is described by the diagrams in Fig.1. The direct diagram must be added to the crossed diagram, in which the photon lines are exchanged [13]. If both diagrams are not added coherently, gauge invariance is seriously violated. This point is frequently mishandled in the literature. We denote the fourmomenta of the leptons by $k$ and $q$. A key role is played by the four-momenta tranferred at the photon-lepton vertices,

$$
\lambda=k-p, \mu=q-p
$$

where $p$ refers to the intermediate fermion line. The timelike components of these vectors have immediate physical interpretations. If $\omega_{a}$ and $\omega_{b}$ are the virtual photon frequeacies in 
the collider frame, then

$$
\lambda_{0}=\omega_{a}, \mu_{0}=\omega_{b}
$$

The dynamics of electromagnetic pair production follow from the Lagrangian for the semiclassical coupling of the electromagnetic field to the lepton density,

$$
\mathcal{L}_{\text {int }}(x)=-\sum_{\ell} \bar{\Psi}_{\ell}(x) \gamma_{\rho} \Psi_{\ell}(x)\left[A_{a}^{\rho}(x)+A_{b}^{\rho}(x)\right]
$$

The lepton number is separately conserved for each generation $\ell$ [10], which we shall consider independently. The four-potential in the Lorentz gauge $A_{a}^{\rho}$ is a classical field derived from the nuclear current $J_{a}^{\rho}$, by the usual relation of electromagnetic theory,

$$
\partial \cdot \partial J_{a}^{\rho}=A_{a}^{\rho}
$$

The classical quantities $A$ and $J$ are functions of time, depending parametrically on the classical trajectories of the ions. Given the Lagrangian (4), we can derive the equations of motion for state vectors in the Schrödinger picture under a series of assumptions, described in detail in our earlier papers [15]. All orders of electromagnetic processes can be obtained from these equations. In general, the elements of the S-matrix connect states which are products of single-particle and antiparticle states, $\chi_{k}^{(+)}$and $\chi_{q}^{(-)}$, satisfying

$$
\left[E_{k}^{(+)}-H_{0}\right] \chi_{k}^{(+)}=0,\left[E_{q}^{(-)}-H_{0}\right] \chi_{q}^{(-)}=0
$$

where

$$
E_{k}^{( \pm)}= \pm\left(1+\vec{k}^{2}\right)^{\frac{1}{2}}
$$

and

$$
H_{0}=\vec{\alpha} \cdot \vec{p}+\gamma_{0}
$$

If we focus on the second-order processes expressed by the diagram in Fig. 1, the relevant S-matrix element is given by [16],

$$
\begin{aligned}
\left\langle f\left|S_{a b}\right| i\right\rangle & =(-i)^{2} \int_{-\infty}^{\infty} d \tau \int_{-\infty}^{\tau} d \tau^{\prime}<f \mid K_{0}(0, \infty) K_{0}(\infty, \tau) \\
& \times V_{a}(\tau) K_{0}\left(\tau, \tau^{\prime}\right) V_{b}\left(\tau^{\prime}\right) K_{0}\left(\tau^{\prime},-\infty\right) K_{0}(-\infty, 0) \mid i>
\end{aligned}
$$


where

$$
|i>=| \chi_{q}^{(-)}>,|f>=| \chi_{k}^{(+)}>.
$$

and $S_{b a}$ is obtained from $S_{a b}$ by reversing the signs of the velocity $\beta$ and the impact paraneter $\vec{\rho}$. The inclusive pair production cross section is given, adequately for the purposes in hand [13], by the expression

$$
\sigma=\int d^{2} \rho \sum_{k>0} \sum_{q<0}|<f|\left|S_{a b}+S_{b a}\right| i>\left.\right|^{2} .
$$

We have only made the approximation of neglecting some small interference terms.

For point charges, the nonzero components of the potential from nucleus $a$, in momentum space and the Lorentz gauge, are given by,

$$
\begin{aligned}
& A^{0}(a)=-8 \pi^{2} Z \gamma^{2} \frac{\delta\left(q_{0}-\beta q_{z}\right)}{q_{x}^{2}+\gamma^{2} q_{\perp}^{2}} \exp \left[i \overrightarrow{q_{\perp}} \cdot \frac{\vec{\rho}}{2}\right] \\
& A^{x}(a)=\beta A^{0}(a) .
\end{aligned}
$$

The potentials from nucleus $b$ can be easily obtained from (12) by the substitutions,

$$
\vec{\rho} \rightarrow-\vec{\rho}, \beta \rightarrow-\beta
$$

We shall later indicate the modifications required for extended charge distributions.

The pair production amplitude for the diagram in Fig. 1 was given in (9). We introduce Fourier representations for the two interactions in the Schrödinger picture,

$$
V_{a}\left(\omega_{a}\right)=-\gamma_{0} \gamma^{\rho} A_{\rho}(a), \quad V_{b}\left(\omega_{b}\right)=-\gamma_{0} \gamma^{\rho} A_{\rho}(b)
$$

and perform the integrations over time, to find that, 


$$
\begin{aligned}
\left\langle f\left|S_{a b}\right| i\right\rangle & =i \int_{-\infty}^{\infty} \frac{d \omega}{2 \pi} \sum_{p} \sum_{a}\left(E_{p}^{(a)}-\omega\right)^{-1} \\
& \left.\times<k\left|V_{a}\left(\omega_{a}\right)\right| p><p\left|V_{b}\left(\omega_{b}\right)\right| q\right\rangle
\end{aligned}
$$

The summation on the intermediate states of $H_{0}$ in (14) is over spin and momentum,

$$
\begin{aligned}
\sum_{p} & =\sum_{\sigma_{p}} \sum_{\vec{p}} \\
& \rightarrow \sum_{\sigma_{p}} \int \frac{d^{3} p}{(2 \pi)^{3}}
\end{aligned}
$$

where the momentum in intermediate states is composed of parts transverse and parallel to the motion of the heavy ions, $\vec{p}=\vec{p}_{\perp}+p_{z} \vec{e}_{z}$. The frequencies $\omega_{a}$ and $\omega_{b}$ are related to the running variable $\omega$ through,

$$
\omega_{a}=E_{k}^{(+)}-\omega, \omega_{b}=\omega-E_{q}^{(-)}
$$

Adding $\omega_{a}+\omega_{b}$ recovers the conservation of energy. The conservation of momentum parallel to the collision axis is expressed by the delta functions in (12),

$$
\begin{aligned}
& k_{z}-p_{z}=\lambda_{z}=\omega_{a} / \beta \\
& q_{z}-p_{z}=\mu_{z}=\omega_{b} / \beta
\end{aligned}
$$

The frequencies and intermediate longitudinal momentum are determined by (15) and (16) as functions of $\beta$,

$$
\begin{aligned}
& p_{z}(\beta)=\frac{-E_{k}^{(+)}+E_{q}^{(-)}+\beta\left(k_{z}+q_{z}\right)}{2 \beta} \\
& \omega(\beta)=\frac{E_{k}^{(+)}+E_{q}^{(-)}-\beta\left(k_{z}-q_{z}\right)}{2}
\end{aligned}
$$

Thus, for fixed momentum and spin states, the transition matrix element in (14) can be written as, 


$$
\begin{aligned}
\left\langle\chi_{k}^{(+)}\left|S_{a b}\right| \chi_{q}^{(-)}\right\rangle & \left.=\frac{i}{2 \beta} \int \frac{d^{2} p_{\perp}}{(2 \pi)^{2}} \exp \left[i \mid \vec{p}_{\perp}-\left(\frac{\vec{k}_{\perp}+\vec{q}_{\perp}}{2}\right)\right] \cdot \vec{\rho}\right] \\
& \times F\left(\vec{k}_{\perp}-\vec{p}_{\perp}: \omega_{a}\right) F\left(\vec{p}_{\perp}-\vec{q}_{\perp}: \omega_{b}\right) \mathcal{T}_{k q}\left(\vec{p}_{\perp}: \beta\right),
\end{aligned}
$$

where $\rho$ denotes the impact parameter, and the function $F$ is the scalar part of the field from each heavy ion. For point charge nuclei, $F$ is given by,

$$
F(\vec{q}: \omega)=\frac{4 \pi Z \gamma^{2} \beta^{2}}{\omega^{2}+\beta^{2} \gamma^{2}|\vec{q}|^{2}}
$$

The function $\mathcal{T}$ explicitly depends on the velocity of the heavy ions $\beta$, on the transverse momentum $\vec{p}_{\perp}$, and on the states $k, q$ : it is given by,

$$
\begin{aligned}
\mathcal{T}_{k q}\left(\vec{p}_{l}: \beta\right) & =\sum_{s} \sum_{\sigma_{p}}\left\{E_{p}^{(s)}-\left[\frac{E_{k}^{(+)}+E_{q}^{(-)}}{2}\right]+\beta\left[\frac{k_{z}-q_{z}}{2}\right]\right\}^{-1} \\
& \times<u_{\sigma_{k}}^{(+)}\left|\left(1-\beta \alpha_{z}\right)\right| u_{\sigma_{p}}^{(0)}>\left\langle u_{\sigma_{p}}^{(0)}\left|\left(1+\beta \alpha_{z}\right)\right| u_{\sigma_{q}}^{(-)}\right\rangle
\end{aligned}
$$

where $u_{\sigma}^{(0)}$ is the spinor part of the states $\chi^{(0)}$. Finally, we note that with the expression for the $S$-matrix (19), the integration on the impact parameter for the pair cross section can be explicitly evaluated. Including both the direct and crossed photon contributions yields the result,

$$
\begin{aligned}
\sigma & =\frac{1}{4 \beta^{2}} \sum_{\sigma_{k} \sigma_{q}} \int \frac{d^{3} k d^{3} q d^{2} p_{\perp}}{(2 \pi)^{8}} \\
& \times\left|\mathcal{A}^{(+)}\left(k, q: \vec{p}_{\perp}\right)+\mathcal{A}^{(-)}\left(k, q: \vec{k}_{\perp}+\vec{q}_{\perp}-\vec{p}_{\perp}\right)\right|^{2}
\end{aligned}
$$

where,

$$
\begin{aligned}
& \mathcal{A}^{(+)}\left(k, q: \vec{p}_{\perp}\right)=F\left(\vec{k}_{\perp}-\vec{p}_{\perp}: \omega_{a}\right) F\left(\vec{p}_{\perp}-\vec{q}_{\perp}: \omega_{b}\right) \mathcal{T}_{k q}\left(\vec{p}_{\perp}:+\beta\right) \\
& \mathcal{A}^{(-)}\left(k, q: \vec{p}_{\perp}\right)=F\left(\vec{k}_{\perp}-\vec{p}_{\perp}: \omega_{b}\right) F\left(\vec{p}_{\perp}-\vec{q}_{\perp}: \omega_{a}\right) \mathcal{T}_{k q}\left(\vec{p}_{\perp}:-\beta\right)
\end{aligned}
$$


The amplitude $\mathcal{T}$, defined in (21), relates the intermediate photon lines to the outgoing fermion lines. It is conveniently recast by summing over the intermediate spinor indices $s, \sigma_{p}$, to find,

$$
\mathcal{T}_{k q}\left(\overrightarrow{p_{\perp}}: \beta\right)=\left\langle u_{\sigma_{k}}^{(+)}\left|\left(1-\beta \alpha_{z}\right) \frac{H_{0}(\vec{p})+\omega}{H_{0}(\vec{p})^{2}-\omega^{2}}\left(1+\beta \alpha_{z}\right)\right| u_{\sigma_{q}}^{(-)}\right\rangle
$$

where,

$$
\begin{aligned}
H_{0}(\vec{p}) & =H_{\perp}\left(\vec{p}_{\perp}\right)+p_{z} \alpha_{z}, \\
H_{\perp}\left(\vec{p}_{\perp}\right) & =\vec{\alpha}_{\perp} \cdot \vec{p}_{\perp}+\gamma_{0},
\end{aligned}
$$

and where $\left(\omega, p_{z}\right)$ depend on $\beta$, as in (18).

In the case of an extended charge distribution, the point charge interactions in momentum space, denoted by $F$ in (19) and (20), are multiplied by the nuclear elastic form factors $G_{E}$. The extension to inelastic nuclear processes has also been worked out [14].

The cross section for lepton production can be evaluated without further approximation using the Monte Carlo techniques described in Ref. [13]. However it is useful to explore some approximate analytic expressions.

\section{Analytic Reductions}

Most approximations to (22) seek to integrate out the transverse momenta $\left(\vec{k}_{\perp}, \vec{p}_{\perp}, \vec{q}_{\perp}\right)$, resulting in an expression for the total cross section of the form [17],

$$
\sigma=\int d k_{z} d q_{z} d^{2} K \Phi\left(\omega_{a}\right) \Phi\left(\omega_{b}\right) \sigma_{\gamma \gamma}\left(\omega_{a}, \omega_{b}: m_{\perp}\right)
$$

where

$$
\vec{K}=\frac{1}{2}\left(\vec{k}_{\perp}+\vec{q}_{\perp}\right)
$$


is the transverse momentum of the pair, and where,

$$
m_{\perp}=\left(1+\vec{K}^{2}\right)^{\frac{1}{2}}
$$

is the transverse mass. To derive (26) from (22), the form factors (20) are integrated over the transverse momentum to obtain "photon fluxes",

$$
\Phi(\omega)=\frac{1}{(2 \pi)^{3} \beta \omega} \int d^{2} \lambda_{\perp} \lambda_{\perp}^{2}\left|F\left(\vec{\lambda}_{\perp}: \omega\right)\right|^{2}
$$

In electron-positron production, the integral must be cut off at $\lambda_{\perp} \simeq m_{\perp}$, but this is unnecessary for the heavier leptons. The nuclear form factor removes momenta $\lambda>1 / R_{\text {nuc }}$, where $R_{\text {nuc }}$ is the nuclear radius in reduced Compton Wavelengths of the lepton in question. Since this limit is more stringent than the former, the integral is simply taken to infinity. For electrons we find that,

$$
\Phi(\omega)=\left(\frac{Z \alpha}{2 \pi \beta}\right)^{2} \frac{1}{\beta \omega} \mathcal{P}\left(\frac{\omega}{\beta \gamma m_{\perp}}\right)
$$

while for heary leptons,

$$
\Phi(\omega)=\left(\frac{Z \alpha}{2 \pi \beta}\right)^{2} \frac{1}{\beta \omega} \mathcal{Q}\left(\frac{\omega R_{n u c}}{\beta \gamma}\right)
$$

The functions $\mathcal{P}$ and $\mathcal{Q}(w)$ are worked out in [13] and [14]; for present purposes, it is enough to note that for large $w$, both $\mathcal{P}, \mathcal{Q}(w) \sim \ln (1 / w)$.

We now consider the remaining factor in (26), the effective two-photon cross section, $\sigma_{\gamma \gamma}$, derived from $T_{k q}$. Many approximations can be formulated: the virtual photon method is not a unique prescription. However we have found that the Two Peak Approximation introduced in [13] very successfully reproduces exact Monte Carlo calculations, including differential cross sections, over a wide range of conditions. This method eliminates the transverse momenta from (22) by observing that the form factors are strongly peaked about the point

$$
\vec{k}_{\perp}, \vec{p}_{\perp}, \vec{q}_{\perp} \simeq \vec{K}
$$

In view of our remark, following (29), this is rendered a better approximation for heavy leptons by the more localized nature of the form factor in momentum space. 
If all frequencies and momenta in $\mathcal{T}$, except the leading powers of $\vec{\lambda}$ and $\vec{\mu}$, are evaluated at the point $\overrightarrow{p_{\perp}}=\vec{K}$, we immediately obtain from (22) an expression for the differential cross section of the form (26),

$$
\begin{aligned}
\frac{d^{4} \sigma}{d k_{z} d q_{z} d^{2} K} & =\frac{8}{\pi^{2} \beta^{4}}(Z \alpha)^{4} \frac{\mathcal{P}\left(\beta \gamma m_{\perp} / \omega_{a}\right) \mathcal{P}\left(\beta \gamma m_{\perp} / \omega_{b}\right)}{\left[m_{\perp}^{2} E_{k}^{(+)} E_{q}^{(-)}\left(1+\mathcal{Z} / \gamma^{2}\right)^{2}\right]^{2}} \\
& \times\left[\mathcal{W}_{0}^{2}+\mathcal{W}_{1}^{2} K^{2}\right] .
\end{aligned}
$$

The rather complicated functions $\mathcal{W}_{0}, \mathcal{W}_{1}$, and $\mathcal{Z}$ of $\left(k_{z}, q_{z}, m_{\perp}\right)$ are defined by the following relations:

$$
\mathcal{Z}=-\frac{1}{4}\left[\frac{Y_{k} Y_{q}}{m_{\perp}^{2}}+\frac{m_{\perp}^{2}}{Y_{k} Y_{q}}\right]
$$

and

$$
\mathcal{W}_{0}=\frac{Y_{k}+Y_{q}}{Y_{k}-Y_{q}}, \quad \mathcal{W}_{1}=\frac{Y_{k}^{2}+Y_{q}^{2}}{\left(Y_{k}-Y_{q}\right)^{2}},
$$

where $Y_{k}$ and $Y_{q}$ are short for,

$$
Y_{k}=E_{k}^{(+)}+k_{z}, \quad Y_{q}=E_{q}^{(-)}+q_{z}
$$

All these quantities are functions of the single transverse mass, $m_{\perp}^{2}=1+\vec{K}^{2}$. We repeat that this is independent of the choice of gauge, if and only if both diagrams are correctly included [18].

Finally, we consider the cross section for the capture process:

$$
a+b \rightarrow a\left(\ell^{-}\right)+b+\ell^{+}(q) .
$$

A gauge invariant result is obtained by folding the matrix element of (22) into the momentum distribution of the final bound state,

$$
<u_{\sigma}^{(s)}(\vec{k})|a, b d\rangle=\phi_{\sigma}^{(s)}(\vec{k}) .
$$

In the Two Peak approximation, we find for capture from a specific vacuum state, (22) into the momentum distribution if the final bound state,

$$
\frac{d^{2} \sigma_{c a p}}{d^{2} q}=\frac{2}{\pi}\left(\frac{\gamma Z \alpha}{\omega}\right)^{2} \sum_{\sigma_{q}}\left|\sum_{\sigma_{k}, s}<u_{\sigma_{q}}^{(s)}(\vec{q})\right| 1+\beta \alpha_{z}\left|u_{\sigma_{k}}^{(\bullet)}(\vec{k})>\phi_{\sigma_{k}}^{(\bullet)}(\vec{k})\right|^{2} .
$$


The single frequency $\omega$ is given by,

$$
\omega=E(a, b d)-E_{q}^{(-)} .
$$

\section{Numerical Results}

We shall present exact calculations of the second order perturbation formula described in Section 2. The integrations in (22) were evaluated by Monte Carlo techniques to an accuracy of about one percent. Details of the numerical method are given elsewhere [13]. Some feeling for the numerical effort involved is conveyed by Fig. 2, which shows the numerical error as a function of the number of Monte Carlo pints. Runs as long as $10^{8}$ points are needed to accumulate statistics for differential cross sections. Extensive tests of the numerical procedures have been carried out, including calculations of the pair production starting from different gauges. Our numerical results can be compared with the two peak formula (33), which appears nearly to reproduce the exact results over a wide range of energies.

In Fig. 3 we show the variation of the total cross section with energy for colliding beams of heavy ions. As previously stated, $\gamma-1$ is the beam kinetic energy per nucleon in units where the nucleon mass is one. The exact results are compared with two currently quoted approximations. The cross section for producing an electron pair from ions of charges $\left(Z_{a}, Z_{b}\right)$ is conveniently expressed in terms of a reduced cross section $\sigma_{0}$,

$$
\sigma_{0}=\lambda^{2} Z_{a}^{2} Z_{b}^{2} \alpha^{4}
$$

where $\lambda=\hbar / m c$ is the reduced Compton wavelength of the electron. For $A u+A u, \sigma_{0}=$ $1.49 \mathrm{~Kb}$. Extrapolating the exact results to infinite $\gamma$ leads to the result,

$$
\sigma \sim C_{\infty} \sigma_{0} \ln (\gamma)^{3}
$$

where, $C_{\infty}=2.19$. The dotted curve is the equivalent photon approximation of Ref. [19], which is essentially a modern version of the Weizsäcker-Williams method with $C_{\infty}=$ 
$224 / 27 \pi \simeq 2.64$. The dot-dashed curve is calculated from the formulae of Ref. [20], which attempt to refine the Weizsäcker-Williams result. It is correct for large $\gamma$, but appears to break down by as much as a factor of ten for $\gamma \leq 20$.

The Two Peak approximation reproduces the exact results to about $15 \%$ over the range of energies per nucleon between $3-300 \mathrm{GeV}$, which will be of most experimental interest in the near future. It has a drawback that it behaves asymptotically as $\ln (\gamma)^{2}$. The origin of this discrepancy is analyzed in Ref [13], though it has little practical significance, for reasons explained in Section 5.

Diff rential cross sections provide more detailed insights. Figures 4 and 5 present angle and momentum distributions in the laboratory for a fixed target experiment at an energy per nucleon of $200 \mathrm{GeV}$. This is equivalent to a colliding beam experiment with $\gamma=10$. Heavyion beams of this energy are presently available at CERN. The differential cross section of the produced electron in terms of the laboratory momentum $\vec{k}$ is obtained from (22) by,

$$
\begin{aligned}
\sigma & =\int_{0}^{\infty} d k \frac{d \sigma}{d \bar{k}} \\
& =\int_{0}^{2 \pi} d \theta \frac{d \sigma}{d \theta}
\end{aligned}
$$

where $\cos (\theta)=k_{z} / k$. The agreement between exact and approximate results, within $20 \%$, is reassuring. The distributions computed in either way are consistent with a picture in which single leptons are ejected with $k_{x} \sim \gamma m c$ and $k_{\perp} \sim m c$; in this case, $\theta \sim 0.3 \mathrm{deg}$ and $k \sim 200 m c$.

The one serious failure of the Two Peak approximation, which is shared by all virtual photon methods, occurs in the differential distribution with respect to the pair mass,

$$
M=\left[\left(k_{0}+q_{0}\right)^{2}-(\vec{k}+\vec{q})^{2}\right]^{\frac{1}{2}} .
$$

Exact calculations show a long tail falling off roughly as $\mathrm{M}^{-4}$, which cannot be described by any virtual photon method $[13,14]$. An accurate knowledge of this regime may be needed to design dilepton experiments, particularly since cuts at large $M^{2}$ are used to enhance hadronic signals. 
To illustrate the size of predicted cross sections for the heavier leptons, we consider $A u+A u$ collisions at $\gamma=10$ and $\gamma=100$. The cross sections for producing $\mu^{ \pm}$pairs at these energies are respectivel $y, 4.2 \mathrm{mb}$ and $178 \mathrm{mb}$, using a realistic nuclear form factor, and the Two Peak approximation. Exact Monte Carlo calculations in progress are giving results in close agreement. The exact point charge result is $645 \mathrm{mb}$.

For $\tau^{ \pm}$we find that the cross sections are negligible $(\ll 1 n b)$ at $\gamma=10$. At $\gamma=100$ however, we find 2.6 $\mu b$. Curiously the contribution from incoherent production by all the protoni: in each nucleus is only $1.7 n b$. From the perspective of RHIC, these cross sections translate into count rates of several muon pairs a minute, and several tauon pairs an hour.

Finally, we mention preliminary calculations on capture in $S+A u$ collisions at a fixed target energy of $200 \mathrm{Gev} /$ nucleon. Capture to the sulfur nucleus is about $2 b$, compared with a loss rate, calculated analogously, of $800 \mathrm{~b}$. With such a ratio of capture to loss, the recombined ions can probably be detected in an experiment at the CERN SPS facility.

\section{Conclusions}

We have seen that lepton pair production by electromagnetic means is a measureable process, and a potentially valuable probe of nuclear form factors at large values of the momentum transfer. A knowledge of the cross sections is also needed in accelerator design, and in the design of other experiments.

What is the status of the perturbation theory used throughout this paper? Caution is necessary, since at least three reasons can be advanced for believing that low order perturbation theory gives an incomplete description of pair production in heavy-ion collisions. (i) The supposedly small parameter $Z \alpha$ is comparable with unity. The asymptotic $\ln (\gamma)^{3}$ dependence of two photon theory cannot be correct. The reasons supporting this statement are very simple, though they are rarely discussed. (ii) The Froissart cross section bound [21] 
is believed to hold for electromagnetic, as well as for all other processes. This imposes a limit on the pair cross section at high energies; namely,

$$
\sigma<\text { const } \times \ln (\gamma)^{2}
$$

(iii) In higher orders, the $\mathrm{N}$-photon contribution to the cross section $\sim \ln (\gamma)^{N+1}$ at least, demonstrating that the perturbation series should be resummed.

On the other hand, we believe that the second order perturbation results can be used heuristically to derive effective Lagrangians which can be solved non-perturbatively. The two photon amplitude for pair production is reproduced, to a good approximation over much of phase space, by the effective Lagrangian involving only products of invariants of the electromagnetic and fermion fields,

$$
\mathcal{L}_{I}=\frac{c_{1}}{m_{\perp}^{2}}\left(\mathcal{E}^{2}-\mathcal{B}^{2}\right) \bar{\psi} \psi+\frac{c_{2}}{m_{\perp}^{2}}(\mathcal{E} \cdot \mathcal{B}) \bar{\psi} \gamma_{5} \psi
$$

where $\mathcal{E}$ is the electric field, $\mathcal{B}$ the magnetic field in the radiation gauge, and $c_{1}$ and $c_{2}$ are constants. The transverse mass

$$
m_{\perp}=m^{2}+p_{\perp}^{2}=m^{2}-\nabla_{\perp}^{2}
$$

is of course an operator, in this context. However, if we make an ansatz for the wavefunction of each vacuum state

$$
\langle\vec{q}| \vec{r}, t>=\Psi(\vec{r}, t)=\psi_{\perp}\left(\overrightarrow{r_{\perp}}, t\right) \psi_{\|}\left(\overrightarrow{r_{\perp}} \mid z, t\right)
$$

and assume that $\psi_{\|}$is a slowly varying function of $\overrightarrow{r_{\perp}}$, we arrive at a set of one dimensional equations for the functions $\psi_{\|}$at some discrete set of $\overrightarrow{r_{\perp}}$. The coupling between different $\psi_{\| \mid}$is expressed though an auxiliary equation for $\psi_{\perp}$, which involves all the $\psi_{\|}$in a self-consistent fashion. Systems with this type of structure are referred to by Applied Mathematicians as one-and-a-half dimensional. They provide a tractable framework for a non-perturbative description of the vacuum.

It is also probable that in many regimes of energy and momentum transfer, perturbation theory is entirely adequate, especially if the particles produced are very heavy. A challenging 
area is that of non-Abelian gauge theories, where even perturbation theory involves delicate considerations of gauge fixing. The simplest example would be the production of $W^{ \pm}$and Higgs bosons in electroweak theory. All these directions are being pursued.

\section{Acknowledgements}

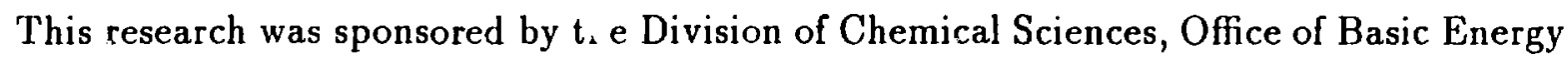
Sciences, and by the Division of Nuclear Physics of the U.S. Department of Energy under contract No. DE-AC05-84OR21400 with Martin Marietta Energy Systems, Inc. 


\section{References}

[1] H. Gould in, "Proceedings of the Atomic Theory Workshop on Relativistic and QED Effects in Heavy Atoms", AIP Conf. Proc. 136, (AIP, New York, 1985).

[2] R. Anholt and H. Gould, in "Advances in Atomic and Molecular Physics", edited by B. Bederson (Academic Press, New York, 1987).

[3] "Physics of Strong Fields", edited by W. Greiner, (Plenum, New York, 1987), Vol. 153, page 1.

[4] E. Teller, in "Proceedings of the Ninth International Conference on the Application of Accelerators in Research and Industry", edited by J.L. Duggan, (North Holland, New York, 1986).

[5] C. Bottcher and M.R. Strayer, Nucl. Inst. and Meth. B31, 122 (1988).

[6] K. Kajantie and H.I. Miettenen, Z. Phys. C9, 341 (1981); Z. Phys. 14, 357 (1982).

[7] K. Kajantie, M. Kataja, L. McLerran, and P.V. Ruuskanen, Phys. Rev. D34, 811 (1986).

[8] K. Kajantie, J. Kapusta, L. McLerran, and A. Mekjian, Phys. Rev. D34, 2746 (1986).

[9] L. McLerran and T. Toimela, Phys. Rev. D31, 545 (1985).

[10] C. Bottcher and M.R. Strayer, in "Physics of Strong Fields", edited by W. Greiner, (Plenum, New York, 1987), Vol.153, page 629.

[11] C. Bottcher and M.R. Strayer, "Proceedings of the Second Workshop on Experiments and Detectors for the RHIC", Berkeley, California, May 25-29, 1987, (LBL-24604).

[12] C. Bottcher and M.R. Strayer, in "Frontiers of Heavy-Ion Physics", edited by N. Cindro, W. Greiner and R. C̆aplar, (World Scientific, Singapore, 1987), page 471. 
[13| C. Bottcher and M. R. Strayer, submitted to Phys. Rev. D

[14] C. Bottcher and M. R. Strayer, submitted to Phys. Rev. D

[15] C. Bottcher and M.R. Strayer, Ann. Phys. (N.Y.) 175, 64 (1987).

[16] M. Baranger and I. Zahed, Phys. Rev. C29, 1005 (1984).

[17] V.B. Berestetskii, E.M. Lifshitz, and L.P. Pitaevskii, "Quantum Electrodynamics", second edition, translated by J.B. Sykes and J.S. Bell, (Pergamon Press, New York, 1979), page 438 .

[18] F. Block and A. Nordsieck, Phys. Rev. 52, 54 (1937).

[19] S. J. Brodsky, T. Kinoshita and H. Terazawa, Phys. Rev. D4, 1532 (1971).

[20] G. Bauer and C.A. Bertulani, Phys. Rev. C35, 836 (1987).

[21] M. Froissart, Phys. Rev. 132, 1053 (1961). 


\section{Figure Captions}

Figure 1: Direct (a), and crossed (b), Feynmann diagrams for pair production in a heavy-ion collision.

Figure 2: Percentage error in Monte Carlo integration scheme, $\Delta$, vs. total number of points accumulated in a series of runs, $N$.

Figure 3: Dependence of pair production cross section with energy. The ratio of the cross section to the reduced cross section defined by (41) is plotted vs. $\gamma$. Full line: exact numerical result; dashed line: equivalent photon approximation [19]; long-short dashed line: modified Weizsäcker-Williams [20].

Figure 4: Angular distribution in the laboratory for a fixed target experiment at an energy per nucleon of $200 \mathrm{GeV}$, equivalent to a colliding beam experiment with $\gamma=10$. The normalized differential cross section (43) is plotted vs. arigle. Full line: exact numerical result; long-short dashed line: two peak approximation (33).

Figure 5: Momentum distribution in the laboratory for a fixed target experiment at an energy per nucleon of $200 \mathrm{GeV}$, equivalent to a colliding beam experiment with $\gamma=10$. The normalized differential cross section (43) is plotted vs. momentum. Full line: exact numerical result; long-short dashed line: two peak approximation (33). 

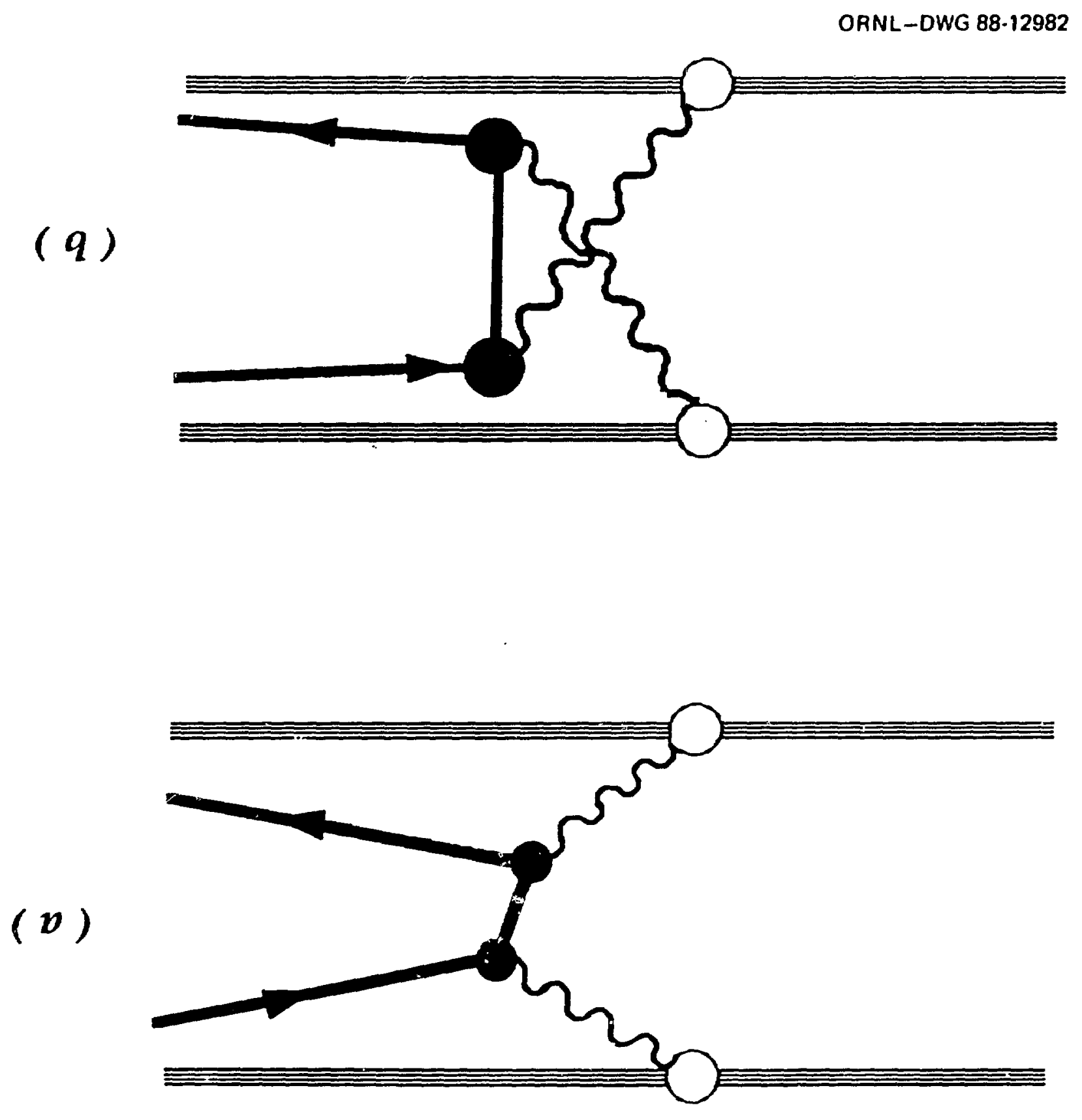


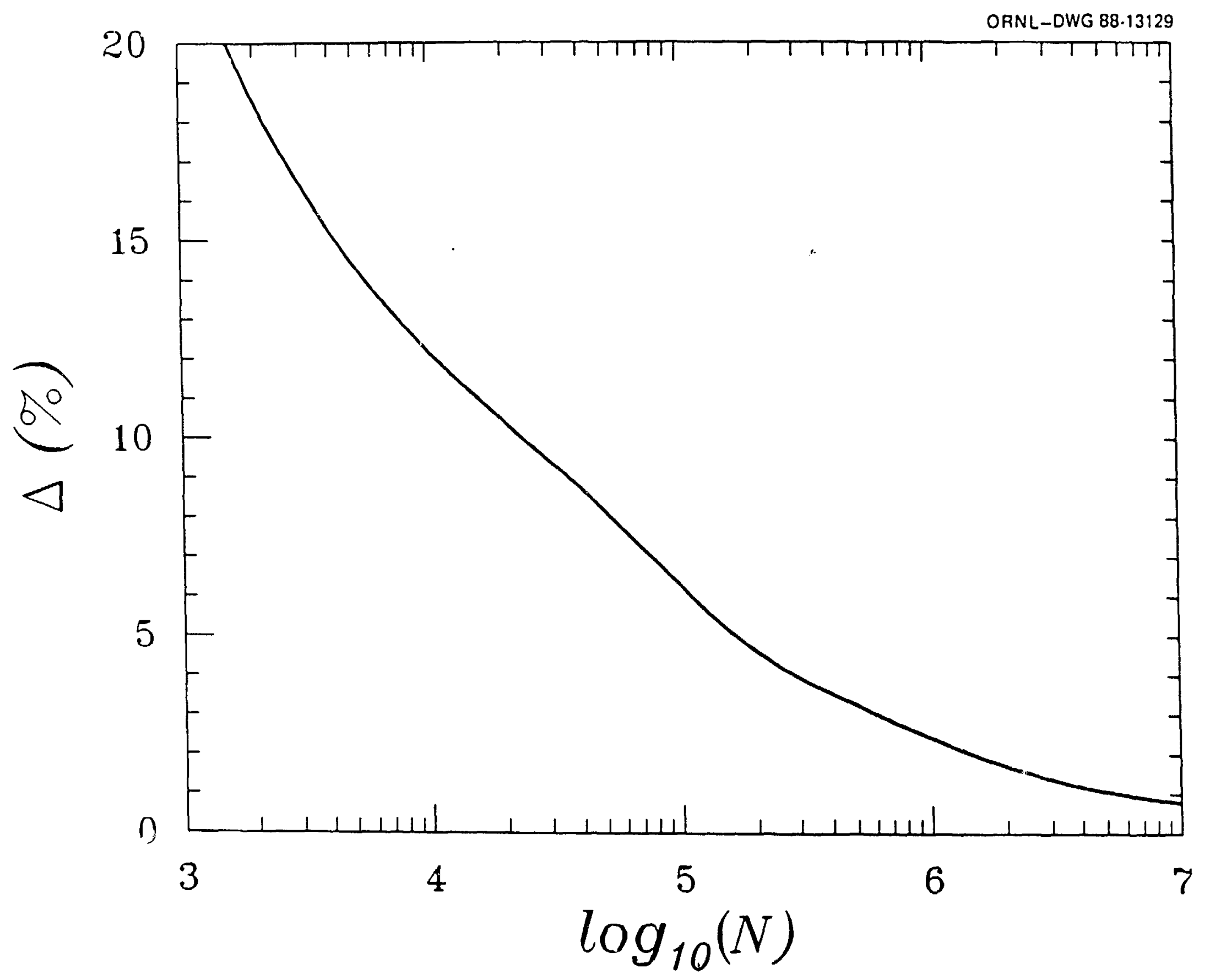




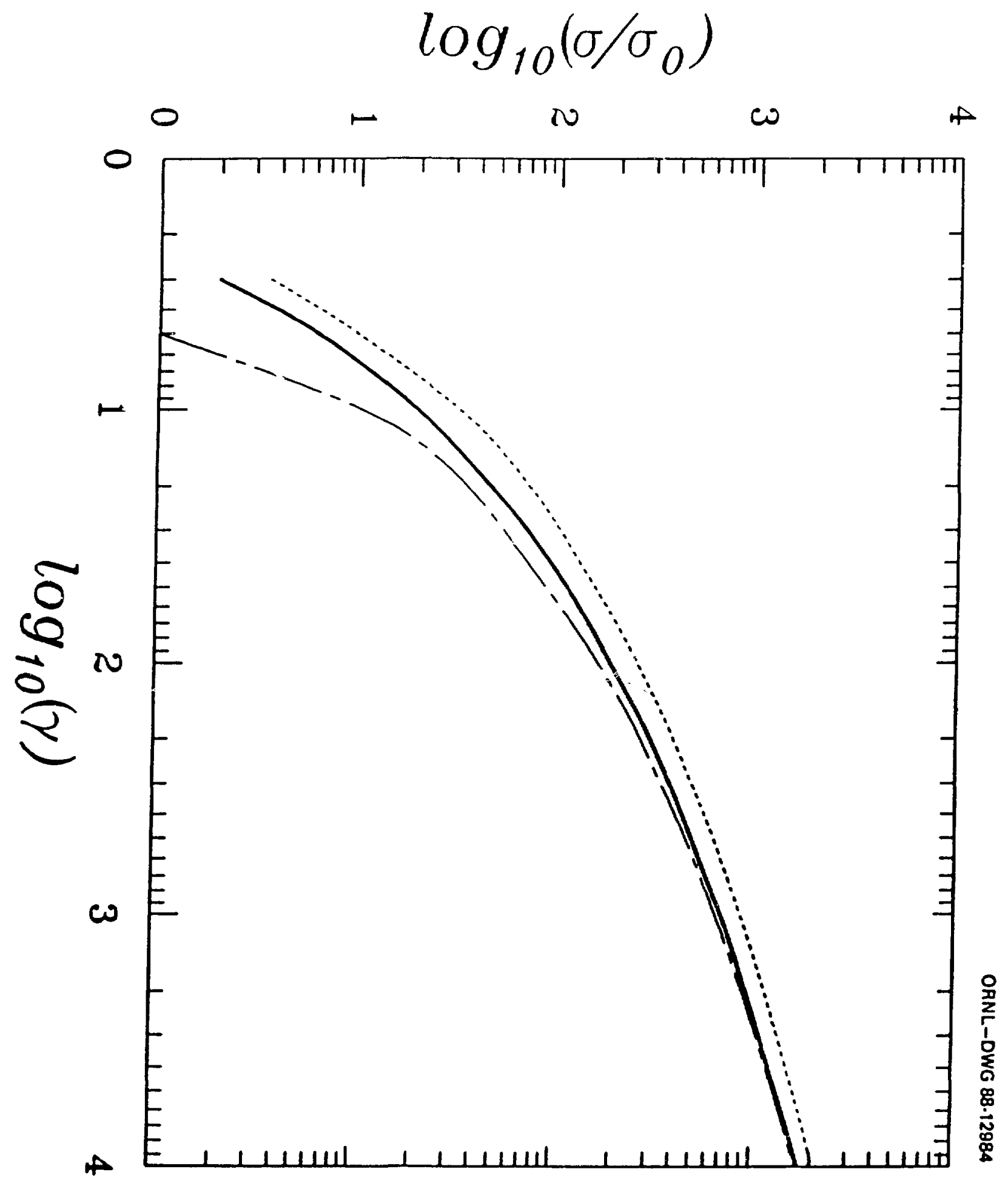

F18. 3 


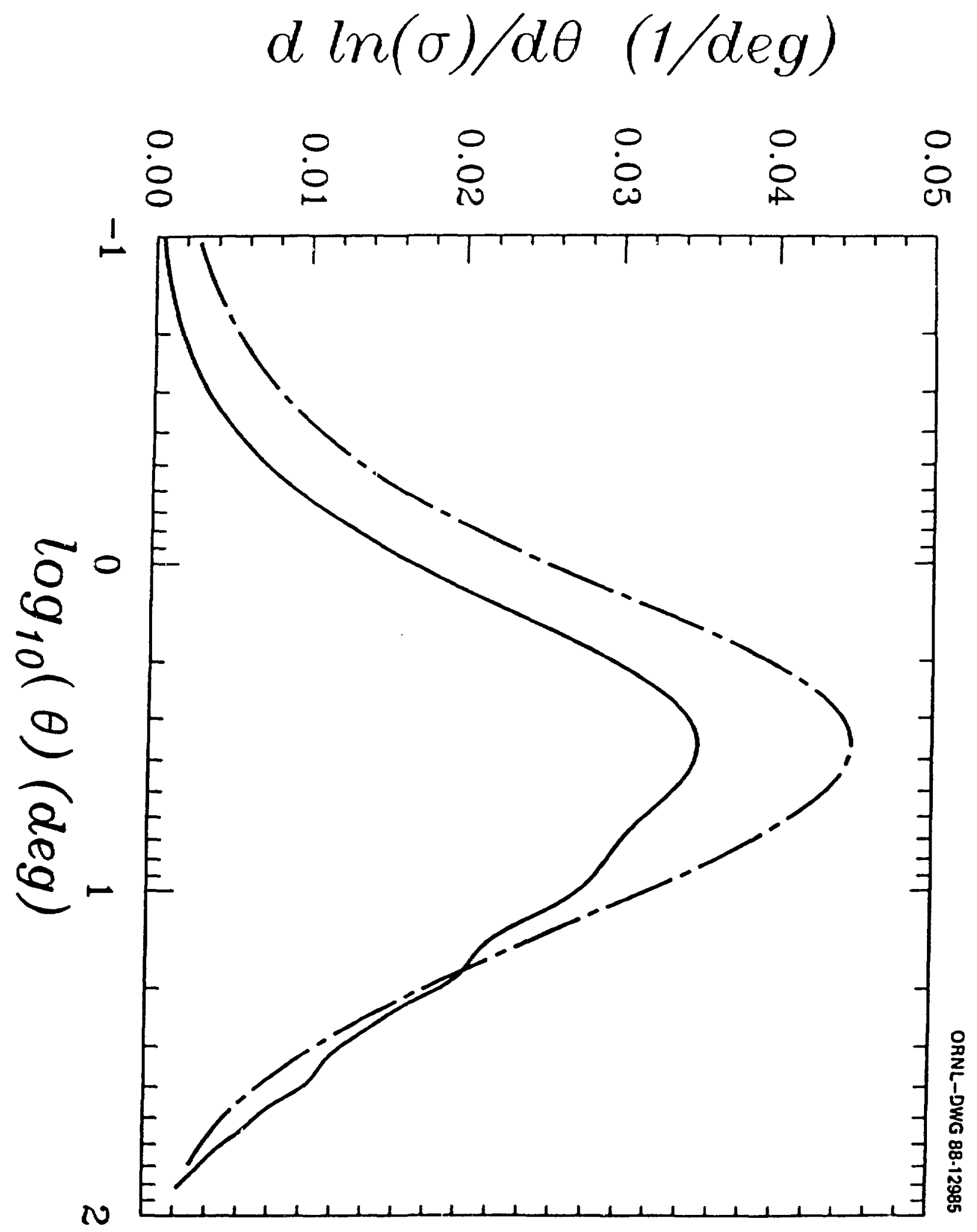

F1g. 4 


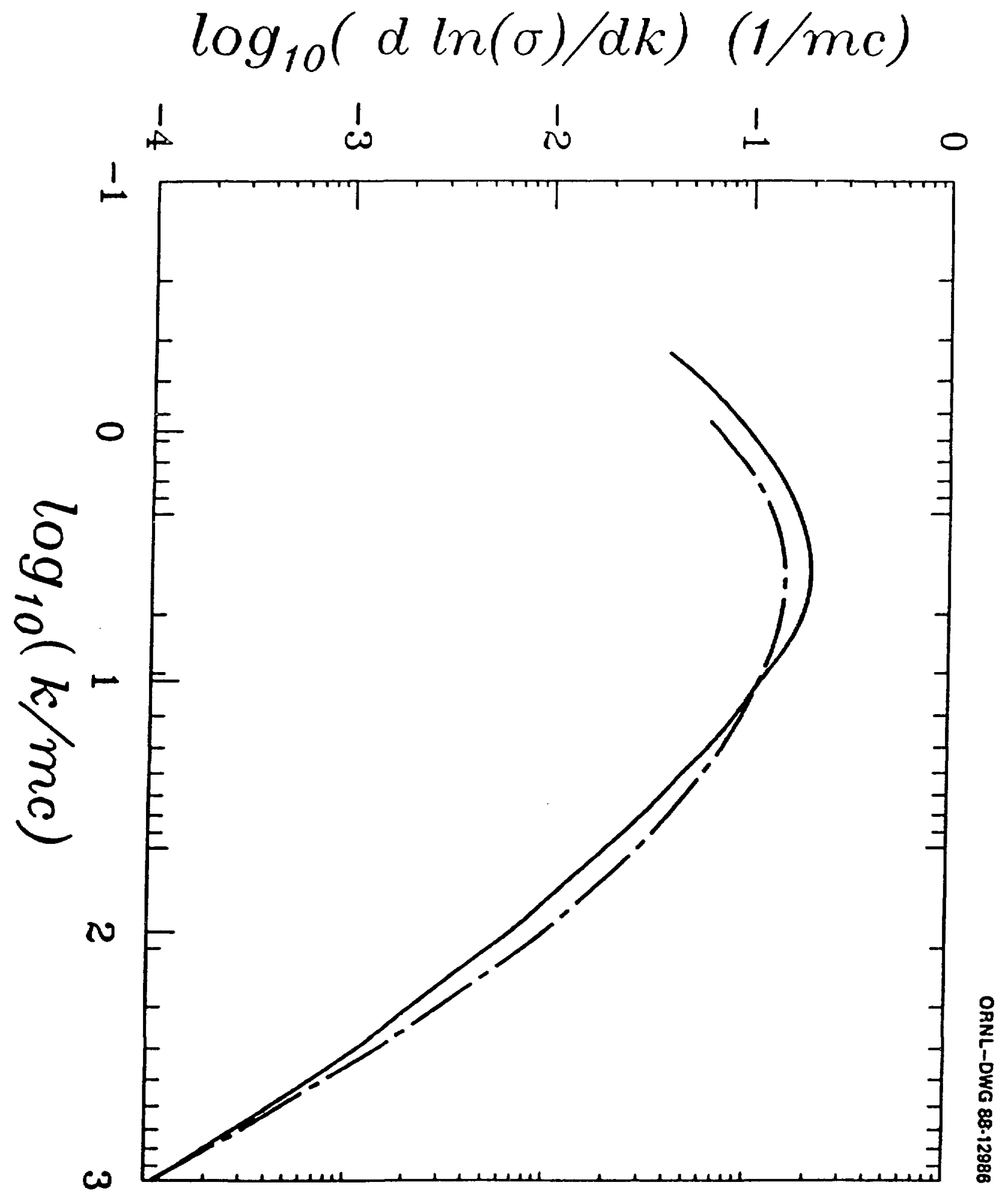

F1g. 5 